PHYSICAL REVIEW E 92, 069903(E) (2015)

\title{
Erratum: Attenuation of excitation decay rate due to collective effect [Phys. Rev. E 90, 022142 (2014)]
}

\author{
B. A. Tay \\ (Received 2 December 2015; published 16 December 2015) \\ PACS number(s): 05.70.Ln, 05.60.-k, 99.10.Cd
}

DOI: 10.1103/PhysRevE.92.069903

There are corrections to a few equations. The corrections do not affect the rest of the discussion and conclusions. In Eqs. (21) and (22) the signs of the $\sin \left(\phi_{0} / 2\right)$ terms should be reversed to read

$$
\begin{aligned}
& A_{1}=a_{1} \cos \left(\phi_{0} / 2\right)-a_{2} \sin \left(\phi_{0} / 2\right), \\
& A_{2}=a_{1} \sin \left(\phi_{0} / 2\right)+a_{2} \cos \left(\phi_{0} / 2\right) .
\end{aligned}
$$

Equations (21) and (22) are now consistent with Eqs. (28) and (29), which involve the inversion of Eqs. (21) and (22).

Equation (27) should read

$$
\begin{aligned}
& \omega_{+} \equiv \omega_{1}^{\prime} \cos ^{2}\left(\phi_{0} / 2\right)+\omega_{2}^{\prime} \sin ^{2}\left(\phi_{0} / 2\right)-J_{12} \sin \phi_{0}, \\
& \omega_{-} \equiv \omega_{1}^{\prime} \sin ^{2}\left(\phi_{0} / 2\right)+\omega_{2}^{\prime} \cos ^{2}\left(\phi_{0} / 2\right)+J_{12} \sin \phi_{0}, \\
& \omega_{ \pm}=\frac{1}{2}\left(\omega_{1}^{\prime}+\omega_{2}^{\prime}\right) \pm \frac{1}{2} \sqrt{\left(\omega_{1}^{\prime}-\omega_{2}^{\prime}\right)^{2}+4 J_{12}^{2}}
\end{aligned}
$$

